\title{
Silk Flow-Diverter Stent for the Treatment of Intracranial Aneurysms: A Series of 58 Patients with Emphasis on Long-Term Results
}

\author{
B. Lubicz, O. Van der Elst, L. Collignon, B. Mine, and F. Alghamdi
}

\begin{abstract}
BACKGROUND AND PURPOSE: The Silk flow-diverter stent is increasingly used to treat complex intracranial aneurysms including wide-neck, fusiform aneurysms. Sparse data are available concerning long-term results of this technique. We report our 5-year experience with Silk stent treatment of intracranial aneurysms.
\end{abstract}

MATERIALS AND METHODS: A retrospective review of our prospectively maintained database identified all patients treated by the Silk stent in 2 institutions. Clinical charts, procedural data, and angiographic results were reviewed.

RESULTS: Between July 2009 and May 2014, we identified 58 patients with 70 intracranial aneurysms. Endovascular treatment was successful in $93 \%$ of patients with 32 treated with the first-generation Silk stent and 26 with the new Silk+ stent. Mean follow-up in 47 patients was 22 months. Despite an $11 \%$ delayed complication rate, overall permanent neurologic morbidity was $5.5 \%$. All complications were seen with the first-generation Silk stent. There was no procedure-related mortality. Long-term anatomic results showed $73 \%$ with complete occlusion, $16 \%$ with neck remnants, and $11 \%$ with incomplete occlusion. No recanalization or retreatment was performed. The midterm intrastent stenosis rate was $57 \%$, of which $60 \%$ improved or disappeared, $28 \%$ were stable, and $12 \%$ led to vessel occlusion. Seventy-four percent of stenosis and all vessel occlusions occurred with the first-generation Silk stent.

CONCLUSIONS: Endovascular treatment of complex intracranial aneurysms with the Silk stent is an effective therapeutic option. Despite a high rate of delayed complications with the first-generation stents, the current Silk + stent appears safer. This treatment achieves a high rate of adequate and stable occlusion at long-term follow-up.

ABBREVIATIONS: CLARITY = Clinical and Anatomical Results in the Treatment of Ruptured Intracranial Aneurysms; PAO = parent artery occlusion

$\mathbf{E}^{2}$

ndovascular treatment is now the first-intention treatment for both ruptured and unruptured intracranial aneurysms. ${ }^{1-4}$ However, endovascular treatment of complex intracranial aneurysms (wide-neck and fusiform) remains technically challenging. On the other hand, aneurysm coil recanalization is an important issue in endovascular therapy, with approximately $20 \%$ of coiled aneurysms recanalized and $10 \%$ retreated. ${ }^{5}$ As previously shown, wide-neck aneurysms are highly prone to recanalization. ${ }^{6}$

These relative limitations of standard coiling have contributed to the development of new endovascular approaches, including balloon-assisted coiling, stent-assisted coiling, flow diversion,

Received July 9, 2014; accepted after revision September 3

From the Department of Neuroradiology (B.L., O.V.d.E., B.M., F.A.), Erasme University Hospital, Brussels, Belgium; and Department of Radiology (L.C.), Centre Hospitalier Régional Citadelle, Liège, Belgium.

Please address correspondence to Boris Lubicz, MD, PhD, Hôpital Erasme, 808 Route de Lennik, 1070 Bruxelles, Belgium; e-mail: blubicz@ulb.ac.be

http://dx.doi.org/10.3174/ajnr.A4143 and flow disruption. Flow-diverter stents have been developed, and they offer the potential of aneurysm occlusion related to flow disruption. Recently, several flow-diverter stents, such as the Silk flow diverter (Balt Extrusion, Montmorency, France), have been released, and short- and midterm results have been published. ${ }^{7-15}$ Notwithstanding the early promise of these devices, important shortcomings have been reported. First, delayed complications, either hemorrhagic or thromboembolic, are not uncommon; second, the flow-diverter stent tolerance might be considered poor because of the relatively high rates of intrastent stenosis and parent artery occlusion (PAO) during follow-up. ${ }^{7-15}$ To the best of our knowledge, sparse data are available about the long-term results of this technique, including stability of aneurysm occlusion and stent tolerance. These data are mandatory to define the place of flow-diverter stents in the therapeutic armamentarium of intracranial aneurysms. Therefore, the aim of our study was to report our 5-year experience with Silk stent treatment of intracranial aneurysms with an emphasis on long-term results. 


\section{MATERIALS AND METHODS}

This retrospective study was approved by our institutional ethics committee. Between July 2009 and May 2014, we retrospectively identified in our prospectively maintained database all patients treated with the Silk stent for 1 or multiple intracranial aneurysms in 2 institutions. All patients underwent conventional angiography of both internal carotid arteries and vertebral arteries. Then, $3 \mathrm{D}$-rotational angiography was performed to depict the aneurysm morphology. These aneurysms were classified as saccular or fusiform and as small ( $<10 \mathrm{~mm}$ ), large (between 10 and $24 \mathrm{~mm}$ ), or giant ( $\geq 25 \mathrm{~mm}$ ). For saccular aneurysms, the neck was classified as wide (neck width of $>4 \mathrm{~mm}$ or dome-to-neck ratio of $<1.6$ ) or small (neck width of $\leq 4 \mathrm{~mm}$ and dome-to-neck ratio of $\geq 1.6$ ).

In all patients, endovascular treatment was performed with the patient under general anesthesia and systemic heparinization. The adequacy of systemic anticoagulation was monitored by repeated measurements of the activated clotting time. A baseline activated clotting time was obtained before the 5000-IU bolus infusion of heparin and hourly thereafter. The bolus infusion was followed by a continuous drip (1500-2500 IU/h) to double the baseline activated clotting time. At the end of the procedure, systemic heparinization was maintained for 24 hours in most patients. All procedures were performed by 2 senior interventional neuroradiologists (B.L., B.M.). The aim of endovascular treatment was to deliver only 1 Silk stent at the aneurysm site. In case of misplacement, a second stent was used to fully cover the aneurysm neck. In all patients, we used a telescopic access system: a long IVA guiding introducer (Balt), a Fargo (Balt) or Neuron (Penumbra, Alameda, California) access catheter, and the delivery Vasco catheter (Silk). From July 2009 to November 2011, the first generation of the Silk stent was used. Then, only the second generation of the stent named "Silk+" was used. In all cases, the Silk stent was first half-deployed and an angiographic control was performed to evaluate the stent tolerance within the parent vessel. If the control was satisfactory, the Silk was fully delivered; if not, the device was removed and other treatments were performed. For planned procedures, a loading dose of $300 \mathrm{mg}$ of clopidogrel and $320 \mathrm{mg}$ of aspirin was administered 1 day before and on the day of endovascular treatment. When stent placement was decided during the procedure, intravenous abciximab was administered a few minutes before stent deployment with a bolus of 0.25 $\mathrm{mg} / \mathrm{kg}$ followed by a continuous perfusion of $0.125 \mu \mathrm{g} / \mathrm{kg} / \mathrm{min}$ for 12 hours. Additional coiling was performed according to the treating physician's judgment. In these cases, a coiling microcatheter was first placed within the aneurysmal sac and then jailed following Silk stent delivery. After endovascular treatment, control DSA was performed, including working, frontal, and lateral views. Patients were then transferred to the intensive care unit; fluid balance, neurologic status, and blood pressure were carefully monitored. In all patients, clopidogrel was maintained for 12 months (75 mg/day), whereas aspirin (160 mg/day) was administered forever. Our antiplatelet treatment remained the same throughout the study.

Procedural and early postprocedural (within 48 hours) complications were recorded. The clinical course was recorded, in- cluding worsening of symptoms and death. Clinical outcome was evaluated according to the modified Rankin Scale. ${ }^{16}$

Patients were evaluated by angiography at the end of the procedure. Anatomic outcome was classified according to the Oxford Grading Scale. ${ }^{17}$

On the basis of clinical chart review, we recorded delayed complications, retreatments, and aneurysm ruptures.

Our imaging follow-up protocol for flow-diverter stents includes DSA every 3 months until aneurysm occlusion. At 12 months (or later if the aneurysm is still not completely occluded), a last DSA and a concurrent MR angiography, which serves as a baseline comparative noninvasive technique, are performed. Then, patients are followed up only by MRA every year (ruptured aneurysms) or every 2 years (unruptured aneurysms). Both timeof-flight and contrast-enhanced MRA are performed. Imaging follow-up duration was defined as the delay between Silk stent placement and the most recent DSA or MRA. We considered follow-up to be short- to-midterm when the latest control examination was performed before 12 months. At $\geq 12$ months, we considered the patient to have undergone a long-term anatomic follow-up.

Two senior neuroradiologists who did not participate in endovascular treatment and 1 medical student (last year of training) reviewed all imaging examinations. Anatomic outcome was classified as complete occlusion (no contrast filling the aneurysm), neck remnant (residual contrast filling the aneurysmal neck), and incomplete occlusion (residual contrast filling the aneurysmal body). Intrastent stenosis was classified as nonsignificant if it was $<50 \%$ and significant or severe if it was $>50 \%$. Parent artery occlusion was also noted. The readers reached a consensus regarding aneurysm occlusion and stent tolerance. The latest available imaging follow-up was compared with the immediate result after endovascular treatment. The evolution of intrastent stenosis was also evaluated and judged stable, improved, or worsened.

\section{RESULTS}

Fifty-eight patients with 70 intracranial aneurysms were identified. There were 40 women and 18 men with a mean age of 48.5 years (range, 14-82 years). Forty-one patients were asymptomatic, whereas 17 were symptomatic. Clinical presentation is detailed in the Table.

Aneurysms were located in the anterior circulation in 59 cases and in the posterior circulation in 11 cases. There were 52 small aneurysms, 17 large aneurysms, and 1 giant aneurysm. Mean aneurysm diameter was $7 \mathrm{~mm}$ (range, 1-46 mm).

There were 51/70 saccular aneurysms, among which 46 had a wide neck. The remaining aneurysms were fusiform $(n=11)$, blood blister-like $(n=6)$, and dissecting $(n=2)$. Two aneurysms were partially thrombosed.

Embolization was successfully performed in 54 patients with 66 aneurysms (93\%), and failure occurred in 4 patients (7\%). In these 4 patients, the Silk stent could not be delivered because of high friction within the delivery microcatheter in 2 cases or because the angiographic controls showed that the stent was not tolerated (nonopacification of the parent artery) in 2 cases. Among these failures, three-fourths occurred with the first-gen- 
No. of Patients

\begin{tabular}{lc}
\hline Asymptomatic & 21 \\
Incidental & 16 \\
Previous SAH & 7 \\
Other IAs to be treated & 4 \\
Post-EVT recanalization & 3 \\
Postclipping remnant & 2 \\
Postclipping regrowth & 3 \\
Familial history & 1 \\
Recanalization & \\
Symptomatic & 9 \\
SAH & 2 \\
WFNS grade 1 & 1 \\
WFNS grade 2 & 1 \\
WFNS grade 3 & 5 \\
WFNS grade 4 & 4 \\
TIA-stroke & 4 \\
Neurologic deficit & 1 \\
Decreased visual acuity & 1 \\
Ophthalmoplegia & 1 \\
Transient tetraparesis & 1 \\
Progressive tetraparesis &
\end{tabular}

Note:-IA indicates intracranial aneurysm; EVT, endovascular treatment; WFNS, World Federation of Neurosurgical Societies.

eration Silk stent. Overall, 32 patients were treated with the Silk stent and 26 with the Silk + stent.

Among 54 treated patients, 48 had only Silk stent placement and 6 had additional coil placement. These 6 patients presented with SAH or a large unruptured aneurysm. All Silk stents could be fully deployed, and no balloon had to be used because no stenosis was encountered. Only 1 technical complication occurred in a patient in whom a cavernous ICA tear occurred due to the access guiding catheter. Two Silk stents were finally placed to occlude the tear, but it failed. Therefore, a test occlusion was performed during endovascular treatment, showing a good tolerance of this ICA occlusion. We stopped heparin, so the aneurysm and the ICA both occluded without any clinical impact.

Clinical complications occurred in 8 patients (8/54, 15\%). Five patients developed a femoral, a retroperitoneal, or cervical hematoma; 1 of these patients experienced a stroke and a hemiparesis due to severe hypotension. Among the 3 remaining patients, 1 had a transient hemiparesis without a clear identifying cause, 1 had a contralateral basal ganglia hematoma 24 hours after uneventful endovascular treatment due to a known severe and uncontrolled hypertension, and 1 had an SAH and a contralateral frontoparietal hematoma a few hours after an uneventful procedure. Among these latter 2 patients, 1 with the basal ganglia hematoma died because of severe intracranial hypertension and the other had no deficit.

Among 54 treated patients (38 asymptomatic, 16 symptomatic), 49 were unchanged, 1 had a severe hemiparesis $(\mathrm{mRS}=4)$, and 4 died. In these latter 4 patients, 3 died because of the initial clinical presentation ( 2 severe SAHs and 1 case of severe pneumonia in a patient who presented with progressive tetraparesis from a giant compressive vertebrobasilar aneurysm) and 1 patient died of intracranial hypertension due to a hypertensive contralateral basal ganglia hematoma. The overall immediate neurologic morbidity rate was thus $1.9 \%$ (1/54). There was no procedure-related mortality.
Among 54 treated patients with 66 aneurysms, immediate aneurysm occlusion was graded 0 in 53 cases $(80.3 \%), 1$ in 6 cases (9.1\%), 2 in 1 case (1.5\%), 3 in 2 cases (3\%), and 4 in 4 cases $(6.1 \%)$. There was 1 parent artery occlusion $(1 / 54,1.9 \%)$. The aneurysms graded 3 and 4 were those treated by the Silk stent and coils.

Among 50 treated patients, clinical and imaging follow-up were available in 47, ranging from 3 to 56 months (mean $=22$ months). Three patients were lost to follow-up. Thirty-six patients had a long-term follow-up, and 11, a short-to-midterm follow-up.

Among 47 patients with follow-up, 5 (5/47, 10.6\%) experienced a delayed thromboembolic complication 11 days to 4 months after endovascular treatment. In 4 cases, the antiplatelet medication was doubled for 12 months, and in 1 patient, an angioplasty was performed to treat a significant intrastent stenosis. Of these patients, 2 kept a slight neurologic deficit ( 1 with $\mathrm{mRS}=$ 1 , and 1 with $\mathrm{mRS}=2$ ) and 3 were completely recovered at follow-up. All of these 5 patients were treated with the first-generation Silk stent. No delayed complications occurred because we used the Silk + stent. There was no aneurysmal rupture.

Overall permanent neurologic morbidity was 5.5\% (1 patient at the immediate phase and 2 at follow-up, $3 / 54$ patients). There was no procedure-related mortality.

Among 47 patients with follow-up with 59 aneurysms, 3 patients with 4 aneurysms experienced an asymptomatic $\mathrm{PAO}(3 / 47$, $6.4 \%$ ). These 3 patients were treated with the first-generation Silk stent and were excluded from the long-term aneurysm occlusion evaluation.

At the latest available imaging examination in 55 aneurysms, 40 were completely occluded (73\%), 9 had a neck remnant (16\%), and 6 were incompletely occluded (11\%). Adequate occlusion (complete + neck remnant) was thus $89 \%$. No recanalization was observed.

At short-to-midterm follow-up in 47 patients, there were 27 intrastent stenoses (57\%). Only 5/27 (18.5\%) stenoses were severe. Twenty stenoses of $27(74 \%)$ occurred in patients treated with the Silk stent, whereas 7 (26\%) occurred in patients treated with the Silk + stent. At long-term follow-up in 25 cases, 15 stenoses improved and/or completely resolved (60\%), 7 were stable (28\%), and 3 asymptomatic PAOs (12\%) occurred. These PAOs were seen in patients treated with the first generation of the Silk stent. In 1 patient with a basilar artery dissection, there was already a significant parent artery stenosis before endovascular treatment. In 1 patient, there was a severe stenosis at short-term follow-up. In the last patient, there was a nonsignificant stenosis at short-term follow-up.

\section{DISCUSSION}

This study shows that endovascular treatment of complex intracranial aneurysms with the Silk stent is an effective therapeutic option. Despite a relatively high rate of delayed complications (thromboembolic event, intrastent stenosis, PAO) with the firstgeneration stents, the current Silk + stent appears safer. Moreover, this endovascular approach achieved a high rate of adequate and stable aneurysm occlusion at long-term follow-up.

This study included all consecutive patients treated in our in- 
stitutions with the Silk stent since its release in 2009 in our country. Despite an obvious learning curve, the rate of successful embolization was very high (93\%). Moreover, 3 of 4 failures occurred with the first-generation Silk stent, which is no longer available. With the new Silk + stent, only 1 failure occurred, suggesting a better stent tolerance at the acute phase of endovascular treatment. The recent introduction of intermediate access catheters (Neuron, Fargo) also helps facilitate the endovascular procedure by adding significant support, for easier and better stent delivery. Our high rate of successful endovascular treatment is in concordance with previous series. ${ }^{7-15}$

It is now generally admitted, and even recommended by the Balt company, that additional coiling be performed in very large or giant unruptured intracranial aneurysms to prevent delayed bleeding. ${ }^{18}$ Our results confirm these findings because we did not observe any bleeding in the present series, with most of our patients having mid-to-long-term follow-up. In our daily practice, we always add coils in ruptured aneurysms and in very large or giant unruptured aneurysms.

The rate of immediate and delayed complications is a major concern with the use of flow-diverter stents. With the first-generation Silk stent, we encountered a high rate of these complications. Since the release of the Silk + stent, none of our patients have experienced a clinical complication. Overall, the long-term clinical follow-up showed a low rate $(5.5 \%)$ of patients with permanent neurologic morbidity and no direct procedure-related mortality. One of our patients died from a contralateral hypertensive hematoma 24 hours after uneventful Silk treatment. We agree that the size of the hematoma was probably increased by the antiplatelet therapy that she had before endovascular treatment, but the severe arterial hypertension was clearly responsible for this complication and not the Silk stent itself. Our good clinical outcome compares favorably with the recent meta-analysis from Murthy et al. ${ }^{14}$ It might be partially explained by the fact that our series included $<25 \%$ of large/giant intracranial aneurysms, whereas Murthy et al included almost two-thirds of these aneurysms. Indeed, the rate of delayed complications is significantly higher in larger intracranial aneurysms in which additional coiling is now recommended. ${ }^{18}$

Another reason might be related to the learning curve with a new device. Indeed, all our permanent complications occurred in our first 20 patients. It is obvious that this technique requires being very familiar with intracranial stent placement. In our referral institutions, 250 intracranial aneurysms are treated by an endovascular approach among which two-thirds are treated with a stent. Therefore, the complete process from the premedication to endovascular treatment and follow-up of patients in the intensive care unit is our daily routine. Last but not least, the Silk stent has been significantly improved with the release of the Silk+ stent, which has flared ends, a higher radial force, and a higher radio-opacity. These features have clearly improved its behavior. Indeed, since we started using the Silk + stent, we have not experienced any clinical complications both during the periprocedural phase and at follow-up.

The present series shows that in this group of complex intracranial aneurysms, adequate occlusion was obtained in a high percentage of cases in long-term results (89\%). Moreover, the treatment was stable with time, with no worsening of the occlusion status. These findings are in concordance with the metaanalysis reported by Murthy et al. ${ }^{14}$ It has been previously shown that neck size is a critical factor for aneurysm recanalization and mid-to-long-term anatomic results in intracranial aneurysms treated with coils.

In the Clinical and Anatomical Results in the Treatment of Ruptured Intracranial Aneurysms (CLARITY) study, aneurysm neck size was identified as the single most important factor in the quality of aneurysm occlusion at midterm follow-up after standard endovascular treatment. ${ }^{4,6}$ In this series, adequate occlusion was obtained in the whole population (narrow and wide-neck aneurysms) and in $80.4 \%$ at midterm follow-up after bare platinum coil treatment. In wide-neck aneurysms, the rate of adequate occlusion was $68.2 \%$, which is much lower than the $89 \%$ observed in the present series. Also in the Ferns et $\mathrm{al}^{5}$ review dealing with narrow and wide-neck aneurysms, the rate of adequate occlusion at follow-up was only $83.4 \%$, lower than the percentage in the present series. With Silk stent treatment, adequate aneurysm occlusion was stable at long-term follow-up and compares favorably with conventional endovascular coiling. Indeed, in CLARITY, worsening of aneurysm occlusion was observed in $51.1 \%$ of aneurysms in the whole group and in $62.1 \%$ of wide-neck aneurysms. Moreover, no retreatment was performed in the present series, and that is a major advantage compared with what was reported in CLARITY (6.2\%) and in the Ferns et al review $(10.3 \%){ }^{4-6}$ These anatomic results are very satisfying because of our selected population that included mostly wide-neck and fusiform intracranial aneurysms.

Stent tolerance is another major concern with flow-diverter stents. Our series showed a very high rate $(57 \%)$ of intrastent stenosis at short- and midterm follow-ups. Most stenoses were asymptomatic, but our rate is significantly superior compared with that in previous series. ${ }^{7-15}$ Indeed, the incidence of intrastent stenosis was $7.8 \%$ in the Berge et al series, ${ }^{10} 6 \%$ in the Byrne et al series, ${ }^{10}$ and $5 \%$ in the Murthy et al meta-analysis. ${ }^{14}$ Nevertheless, the only series that is almost comparable with ours is that of Cohen et $\mathrm{al},{ }^{15}$ which was also focused on this precise evaluation. In this latter study, the rate of intrastent stenosis at the first follow-up examination was $38 \%$. A possible explanation is that we asked 2 neuroradiologists who were not involved in endovascular treatment to evaluate our results to avoid any underestimation. Most interesting, $76 \%$ of these stenoses were seen with the use of the first-generation stents. These anatomic findings are well-correlated to our clinical results, which are much better with the Silk+ stent. Even more important is that these stenoses improved or disappeared in $60 \%$ of cases. This phenomenon has previously been reported, ${ }^{10}$ but our series, with a large number of patients having long-term follow-up until 56 months, brings additional data to highlight the remarkable nature of these stenoses. This particular behavior of improving/disappearing intrastent stenosis should now be better understood by more fundamental research. Nevertheless, this information is of great interest when physicians are facing such problems. Last but not least, PAOs were also seen in the present series, and all occurred with the first-generation stents. Risk factors for PAO are not very well-identified, but preexisting stenosis seems to be one of them. ${ }^{7-15}$ Larger series with 
long-term follow-up are needed to better define patients who are at risk of such pejorative evolution.

Our study has several limitations. First, it was retrospective with a small number of patients. However, all patients were consecutively included and analyzed. Second, imaging follow-up was incompletely homogeneous because it included MRA and DSA. Indeed, MRA has recently been reported to be reliable for the follow-up of intracranial aneurysms treated with flow-diverter stents, but it underestimates the luminal diameter of the reconstructed artery compared with DSA. ${ }^{19}$ However, in our imaging protocol, contemporaneous DSA and MRA are performed when the aneurysm is completely occluded, and these examinations serve as the baseline study for the follow-up. Therefore, the following MRA examinations are compared with the baseline study to detect a decrease of the parent artery diameter.

\section{CONCLUSIONS}

This series shows that endovascular treatment of complex intracranial aneurysms with the Silk stent is effective and is associated with an acceptable neurologic morbidity. Despite a relatively high rate of delayed complications (thromboembolic event, intrastent stenosis, PAO) with the first-generation stents, the current Silk+ stent appears safer. Moreover, this endovascular approach achieves a high rate of adequate and stable aneurysm occlusion at long-term follow-up.

Disclosures: Boris Lubicz-UNRELATED: Consultancy: MicroVention, Codman, Covidien, Sequent.

\section{REFERENCES}

1. Molyneux A, Kerr R, Stratton I, et al; International Subarachnoid Aneurysm Trial (ISAT) Collaborative Group. International Subarachnoid Aneurysm Trial (ISAT) of neurosurgical clipping versus endovascular coiling in 2143 patients with ruptured intracranial aneurysms: a randomised trial. Lancet 2002;360:1267-74

2. McDougall CG, Spetzler RF, Zabramski JM, et al. The Barrow Ruptured Aneurysm Trial. J Neurosurg 2012;116:135-44

3. Cognard C, Pierot L, Anxionnat R, et al; Clarity Study Group. Results of embolization used as the first treatment choice in a consecutive non selected population of ruptured aneurysms: clinical results of the Clarity GDC study. Neurosurgery 2011;69:837-41

4. Pierot L, Spelle L, Vitry F; ATENA Investigators. Immediate clinical outcome of patients harbouring unruptured intracranial aneu- rysms treated by endovascular approach: results of the ATENA study. Stroke 2008;39:2497-504

5. Ferns SP, Sprengers MES, van Rooij WJ, et al. Coiling of intracranial aneurysms: a systematic review on initial occlusion and reopening and retreatment rates. Stroke 2009;40:e523-29

6. Pierot L, Cognard C, Anxionnat R, et al; CLARITY Investigators. Endovascular treatment of ruptured intracranial aneurysms: factors affecting mid-term quality anatomic results: analysis in a prospective multicenter series of patients (CLARITY). AJNR Am J Neuroradiol 2012;33:1475-80

7. Lubicz B, Collignon L, Raphaeli G, et al. Flow-diverter stent for the endovascular treatment of intracranial aneurysms: a prospective study in 29 patients with 34 aneurysms. Stroke 2010;41:2247-53

8. Byrne JV, Beltechi R, Yarnold JA, et al. Early experience in the treatment of intra-cranial aneurysms by endovascular flow diversion: a multicentre prospective study. PloS One 2010;5:pii: e12492

9. Tähtinen OI, Manninen HI, Vanninen RL, et al. The Silk flow-diverting stent in the endovascular treatment of complex intracranial aneurysms: technical aspects and midterm results in 24 consecutive patients. Neurosurgery 2012;70:617-23

10. Berge J, Biondi A, Machi P, et al. Flow-diverter Silk stent for the treatment of intracranial aneurysms: 1-year follow-up in a multicenter study. AJNR Am J Neuroradiol 2012;33:1150-55

11. Wagner A, Cortsen M, Hauerberg J, et al. Treatment of intracranial aneurysms: reconstruction of the parent artery with flow-diverting (Silk) stent. Neuroradiology 2012;54:709-18

12. Velioglu M, Kizilkilic O, Selcuk H, et al. Early and midterm results of complex cerebral aneurysms treated with Silk stent. Neuroradiology 2012;54:1355-65

13. Cirillo L, Leonardi M, Dall'olio M, et al. Complications in the treatment of intracranial aneurysms with Silk stents: an analysis of $\mathbf{3 0}$ consecutive patients. Interv Neuroradiol 2012;18:413-25

14. Murthy SB, Shah S, Shastri A, et al. The Silk flow diverter in the treatment of intracranial aneurysms. J Clin Neurosci 2014;21: 203-06

15. Cohen JE, Gomori JM, Moscovici S, et al. Delayed complications after flow-diverter stenting: reactive in-stent stenosis and creeping stents. J Clin Neurosci 2014;21:1116-22

16. Bonita R, Beaglehole R. Modification of Rankin scale: recovery of motor function after stroke. Stroke 1988;19:1497-500

17. Kamran M, Yarnold J, Grunwald IQ, et al. Assessment of angiographic outcomes after flow diversion treatment of intracranial aneurysms: a new grading schema. Neuroradiology 2011;53:501-08

18. Kulcsár Z, Houdart E, Bonafé A, et al. Intra-aneurysmal thrombosis as a possible cause of delayed aneurysm rupture after flow-diversion treatment. AJNR Am J Neuroradiol 2011;32:20-25

19. Boddu SR, Tong FC, Dehkharghani S, et al. Contrast-enhanced timeresolved MRA for follow-up of intracranial aneurysms treated with the Pipeline embolization device. AJNR Am J Neuroradiol 2014;35: 2112-18 